\title{
Un théorème de disjonction de systèmes dynamiques et une généralisation du théorème ergodique de Wiener-Wintner
}

\author{
E. LESIGNE \\ Université de Bretagne Occidentale, 6 avenue Victor Le Gorgeu, \\ 29287-Brest Cedex, France
}

(Received 22 February 1988)

Résumé. Si $(\Omega, \mathscr{C}, \mu, T)$ est un espace dynamique probabilisé et si $f \in L^{1}(\mu)$, l'ensemble des points $\omega$ pour lesquels la suite

$$
\frac{1}{N} \sum_{n=0}^{N-1}\left\{\exp [i P(n)] \cdot f\left(T^{n} \omega\right)\right\}
$$

converge pour tout polynôme réel $P$, est de mesure 1 .

Pour démontrer ce résultat on utilise la notion de disjonction de systèmes dynamiques et le fait que la disjonction est une propriété préservée par les extensions isométriques.

Abstract. If $(\Omega, \mathscr{C}, \mu, T)$ is a measure preserving system and if $f \in L^{1}(\mu)$ then, for almost all $\omega$, for every real polynomial $P$, the sequence

$$
\frac{1}{N} \sum_{n=0}^{N-1}\left\{\exp [i P(n)] \cdot f\left(T^{n} \omega\right)\right\} \text { converges. }
$$

To prove this result we use the concept of disjointness of dynamical systems and the fact that disjointness is preserved by isometric extensions.

\section{Introduction}

Soit $(\Omega, \mathscr{C}, \mu)$ un espace probabilisé muni d'une transformation $T$ préservant la mesure $\mu$. Le théorème ergodique de Wiener-Wintner [7] affirme que, pour toute fonction $f$ intégrable sur $(\Omega, \mathscr{C}, \mu)$, il existe $\Omega_{f}$ dans la tribu $\mathscr{C}$ tel que: $\mu\left(\Omega_{f}\right)=1$ et pour tout $\omega \in \Omega_{f}$, pour tout $\alpha$ réel, la suite

$$
\left(\frac{1}{N} \sum_{n=0}^{N-1}\left\{\exp [2 \pi i n \alpha] \cdot f\left(T^{n} \omega\right)\right\}\right)
$$

est convergente.

La nouveauté de cet énoncé par rapport au théorème ergodique de Birkhoff réside dans le fait que l'ensemble $\Omega_{\boldsymbol{f}}$ peut être choisi indépendamment du réel $\alpha$.

Nous nous proposons de démontrer le résultat suivant. 
Théorème 1. Pour toute fonction f intégrable sur $(\Omega, \mathscr{C}, \mu)$, il existe $\Omega_{f}^{\prime}$ dans la tribu $\mathscr{C}$ tel que:

$$
\mu\left(\Omega_{j}^{\prime}\right)=1
$$

et pour tout $\omega \in \Omega_{f}^{\prime}$, pour tout $k$ entier positif, pour tous $\alpha_{1}, \alpha_{2}, \ldots, \alpha_{k}$ réels, la suite

$$
\left(\frac{1}{N} \sum_{n=0}^{N-1}\left\{\exp \left[2 \pi i\left(n \alpha_{1}+n^{2} \alpha_{2}+\cdots+n^{k} \alpha_{k}\right)\right] \cdot f\left(T^{n} \omega\right)\right\}\right)
$$

est convergente.

On obtiendra également des résultats sur la limite de la suite (1). En particulier, si le système dynamique $(\Omega, \mathscr{C}, \mu, T)$ est faiblement mélangeant et si $f \in L^{1}(\mu)$, il existe $\Omega_{f}^{\prime}$ de mesure 1 tel que: si $\omega \in \Omega_{f}^{\prime}$ et si un des $\alpha_{j}$ est irrationnel, alors

$$
\lim _{N \rightarrow+\infty} \frac{1}{N} \sum_{n=0}^{N-1}\left\{\exp \left[2 \pi i\left(n \alpha_{1}+n^{2} \alpha_{2}+\cdots+n^{k} \alpha_{k}\right)\right] \cdot f\left(T^{n} \omega\right)\right\}=0 .
$$

\section{Présentation de la démonstration}

Le théorème ergodique de Wiener-Wintner peut se démontrer de façon rapide en utilisant la théorie des systèmes dynamiques disjoints, développée par Furstenberg dans [3] (les définitions seront rappelées dans I.1). Cette démonstration est présentée dans [5]. Elle est basée sur le fait qu'un système dynamique ergodique quelconque et un système dynamique ergodique à spectre discret sont disjoints dès qu'ils n'ont aucune valeur propre en commun. Cette idée et la lecture de [2] et [3] conduisent naturellement à une généralisation du théorème de Wiener-Wintner. Dans [2] et [4], Furstenberg construit des systèmes à spectre discret généralisé pour faire apparaître des expressions de la forme

$$
\frac{1}{N} \sum_{n=0}^{N-1} \exp \left[2 i \pi\left(n \alpha_{1}+n^{2} \alpha_{2}+\cdots+n^{k} \alpha_{k}\right)\right]
$$

et pour démontrer le théorème d'équirépartition de H. Weyl. Dans [3], Furstenberg donne les arguments permettant de démontrer que 'la disjonction est préservée par extension isométrique'. On en déduira qu'un système dynamique ergodique quelconque et un système dynamique ergodique à spectre discret généralisé sont disjoints dès qu'ils n'ont aucune valeur propre en commun.

Tout cela est précisé dans la suite de cette note.

Dans la première partie, on rappelle la définition de la disjonction, la définition des extensions isométriques et on démontre que la disjonction est préservée par extensions isométriques (Théorème 3 ).

Dans la seconde partie, on démontre le Théorème 1. Cette démonstration se décompose en deux parties:

-le Théorème 3 permet d'étudier la convergence presque sûre de (1) pour tous les $k$-uplets $\left(\alpha_{1}, \alpha_{2}, \ldots, \alpha_{k}\right)$ sauf pour une famille dénombrable de valeurs de $\alpha_{k}$; ceci conduit à l'énoncé du Théorème 4 .

-il faut ensuite étudier la convergence presque sûre de (1) pour tous les $k$-uplets $\left(\alpha_{1}, \alpha_{2}, \ldots, \alpha_{k}\right)$ où $\alpha_{k}$ est fixé (voir la remarque du $\$ 2.2$ et la Proposition 2). 
Remarque. Dans toute la suite, on appellera système dynamique un triplet $(\Omega, \mu, T)$ où $\Omega$ est un espace compact métrisable muni de sa tribu borélienne, $\mu$ est une probabilité régulière sur $\Omega$ et $T$ est une transformation continue de $\Omega$, préservant la mesure $\mu$. Grâce à des théorèmes de représentation on sait que cette hypothèse topologique n'est pas réellement restrictive pour les résultats qui nous intéressent (cf. [4], chapitre 5).

\section{Un théorème de disjonction}

\subsection{Systèmes dynamiques disjoints}

Définition 1. Soient $\mathscr{S}=(\Omega, \mu, T)$ et $\mathscr{S}^{\prime}=\left(\Omega^{\prime}, \mu^{\prime}, T^{\prime}\right)$ deux systèmes dynamiques. On appelle couplage des systèmes $\mathscr{S}$ et $\mathscr{S}^{\prime}$ toute mesure régulière sur le produit $\Omega \times \Omega^{\prime}$ qui se projette sur, respectivement, $\mu$ et $\mu^{\prime}$ et qui est invariante par la transformation $T \times T^{\prime}$. On dit que les systèmes $\mathscr{S}$ et $\mathscr{S}^{\prime}$ sont disjoints si la mesure produit $\mu \otimes \mu^{\prime}$ est le seul couplage des deux systèmes.

Rappelons qu'un point $\omega$ du système $\mathscr{S}=(\Omega, \mu, T)$ est dit générique dans $\mathscr{S}$ si, pour toute fonction continue $f$ sur $\Omega$, on a:

$$
\lim _{N \rightarrow+\infty} \frac{1}{N} \sum_{n=0}^{N-1} f\left(T^{n} \omega\right)=\int_{\Omega} f d \mu \text {. }
$$

On sait que si le système $\mathscr{S}$ est ergodique, presque tout point de $\Omega$ est générique dans $\mathscr{S}$; ceci est une conséquence immédiate du théorème ergodique de Birkhoff et de la séparabilité de l'espace des fonctions continues sur $\Omega$, muni de la topologie de la convergence uniforme.

Nous aurons besoin par la suite du résultat suivant, dont la démonstration ne présente pas de difficulté.

THÉORÈmE 2. ([3], Théorème 1.6.) Soient $\mathscr{S}$ et $\mathscr{S}^{\prime}$ deux systèmes dynamiques disjoints. Si $\omega$ est un point générique dans $\mathscr{S}$ et $\omega^{\prime}$ un point générique dans $\mathscr{S}^{\prime}$ alors le couple $\left(\omega, \omega^{\prime}\right)$ est générique dans le système produit $\mathscr{S} \times \mathscr{S}^{\prime}$.

\subsection{Extensions isométriques}

On reprend la définition des extensions isométriques donnée par Thouvenot dans [6], en imposant en plus la continuité des transformations.

Définition 2. Soit $\mathscr{S}_{1}=\left(\Omega_{1}, \mu_{1}, T_{1}\right)$ un système dynamique. Soient $H$ un groupe compact métrisable et $K$ un sous-groupe fermé de $H$. Notons $m$ l'image, sur le quotient $H / K$, de la probabilité de Haar de $H$. Soit $\varphi$ une application continue de $\Omega$ dans $H$. Le système $\mathscr{S}_{2}=\left(\Omega_{2}, \mu_{2}, T_{2}\right)$ défini par $\Omega_{2}=\Omega_{1} \times H / K, \mu_{2}=\mu_{1} \otimes m$ et $T_{2}\left(\omega_{1}, h \cdot K\right)=\left(T_{1} \omega_{1},\left(\varphi\left(\omega_{1}\right) \cdot h\right) \cdot K\right)$, sera appelé une extension isométrique du système $\mathscr{S}_{1}$.

\subsection{La disjonction est conservée par extension isométrique \\ Ce titre est précisé par l'énoncé suivant:}

ThÉORème 3. Soient $\mathscr{S}$ et $\mathscr{S}_{1}$ deux systèmes dynamiques ergodiques. Soit $\mathscr{S}_{2}$ une extension isométrique de $\mathscr{S}_{1}$. Si $\mathscr{S}$ et $\mathscr{S}_{1}$ sont disjoints et si le système produit $\mathscr{S} \times \mathscr{S}_{2}$ est ergodique, alors $\mathscr{S}$ et $\mathscr{S}_{2}$ sont disjoints. 
La preuve de ce théorème est essentiellement contenue dans celle du théorème 1.4 de [3]. La seule nuance est que, dans le cas d'une extension isométrique sous la forme ici définie, l'action de $H$ sur $\Omega_{2}$ ne commute pas nécessairement avec la transformation $T_{2}$.

\section{Preuve du Théorème 3}

On note $\mathscr{S}=(\Omega, \mu, T)$ et on reprend les notations de la Définition 2. Soit $\nu$ un couplage des systèmes $\mathscr{S}$ et $\mathscr{S}_{2}$. Si $\Psi$ est une fonction mesurable, bornée et positive sur $H$, on définit une mesure $\nu_{\Psi}$ sur le produit $\Omega \times \Omega_{2}$ par

$$
\begin{aligned}
& \int f\left(\omega, \omega_{1}, h \cdot K\right) d \nu_{\Psi}\left(\omega, \omega_{1}, h \cdot K\right) \\
& =\iint f\left(\omega, \omega_{1}, h^{\prime} h \cdot K\right) \cdot \Psi\left(h^{\prime}\right) d \nu\left(\omega, \omega_{1}, h \cdot K\right) d h^{\prime},
\end{aligned}
$$

pour toute fonction $f$ continue sur $\Omega \times \Omega_{2}$. (où $d h^{\prime}$ désigne la mesure de Haar normalisée de $H$ ). On va construire une suite $\left(\nu_{\Psi_{n}}\right)$ de 'régularisées' de $\nu$ qui seront absolument continues par rapport à la mesure produit $\mu \otimes \mu_{2}, T \times T_{2}$-invariantes et qui convergeront vers $\nu$. Notons $\lambda$ la projection de $\nu$ sur $\Omega \times \Omega_{1}$. La probabilité $\lambda$ est un couplage de $\mathscr{S}$ et $\mathscr{S}_{1}$ et, ces deux systèmes étant supposés disjoints, on a $\lambda=\mu \otimes \mu_{1}$. La probabilité $\nu_{1}$ (i.e. $\nu_{\Psi}$ avec $\Psi \equiv 1$ ) est égale à $\lambda \otimes m=\mu \otimes \mu_{1} \otimes m=$ $\mu \otimes \mu_{2}$. De plus il est immédiat que, pour toute fonction $\Psi$ mesurable et bornée, la mesure $\nu_{\Psi}$ est absolument continue par rapport à $\nu_{1}$.

Supposons que $\Psi$ est invariante sous les automorphismes intérieurs de $H$, c'est-àdire que, pour tous $h$ et $h^{\prime}$ dans $H, \Psi\left(h h^{\prime} h^{-1}\right)=\Psi\left(h^{\prime}\right)$; montrons qu'alors la mesure $\nu_{\Psi}$ est $T \times T_{2}$-invariante.

$$
\begin{aligned}
\int f \circ( & \left.T \times T_{2}\right) d \nu_{\Psi} \\
= & \int f\left(T \omega, T_{1} \omega_{1}, \varphi\left(\omega_{1}\right) h^{\prime} h K\right) \cdot \Psi\left(h^{\prime}\right) d h^{\prime} d \nu\left(\omega, \omega_{1}, h K\right) \\
= & \int f\left(T \omega, T_{1} \omega_{1}, \varphi\left(\omega_{1}\right) h h^{\prime \prime} K\right) \cdot \Psi\left(h^{\prime \prime}\right) d h^{\prime \prime} d \nu\left(\omega, \omega_{1}, h K\right) .
\end{aligned}
$$

(On a effectué le changement de variable $h^{\prime \prime}=h^{-1} h^{\prime} h$ ). Du fait que $\nu$ est $T \times$ $T_{2}$-invariante, on déduit alors que

$$
\int f \circ\left(T \times T_{2}\right) d \nu_{\Psi}=\int f d \nu_{\psi} .
$$

On a:

$-\nu_{\Psi} \ll \nu_{1}$

- $\nu_{1}$ est $T \times T_{2}$-ergodique (par hypothèse)

- $\nu_{4}$ est $T \times T_{2}$-invariante.

On en déduit que la mesure $\nu_{\psi}$ est proportionnelle à la probabilité $\nu_{1}$. 
Soit $d$ une distance bi-invariante sur le groupe $H$; notons $e$ l'élément neutre de $H$. Posons, pour tout $h$ dans $H$,

$$
\gamma_{n}(h)=\max \left(0, \frac{1}{n}-d(e, h)\right)
$$

Posons

$$
\Psi_{n}=\left(\int_{H} \gamma_{n}(h) d h\right)^{-1} \cdot \gamma_{n} .
$$

Chaque fonction $\Psi_{n}$ est mesurable, bornée, positive et invariante sous les automorphismes intérieurs de $H$. De plus chaque mesure $\nu_{\Psi_{n}}$ est une probabilité. D'après ce qui précède, on a donc $\nu_{\Psi_{n}}=\nu_{1}$.

Du fait que le support de $\Psi_{n}$ est inclus dans la boule centrée en $e$ et de rayon $1 / n$, on déduit que la suite $\left(\nu_{\Psi_{1}}\right)$ converge étroitement vers $\nu$. On peut conclure que $\nu$ est égale à la mesure produit $\nu_{1}$, et le Théorème 3 est démontré.

\section{Un théorème ergodique du type Wiener-Wintner}

L'étude qui précède va nous permettre de démontrer le résultat suivant:

ThÉORÈme 4. Soit $\mathscr{S}=(\Omega, \mu, T)$ un système dynamique ergodique. Soient $\alpha_{1}, \alpha_{2}, \ldots, \alpha_{k}$ des nombres réels. On suppose qu'il existe un indice l entre 1 et $k$ tel que:

(a) $\alpha_{t}$ est irrationnel et $\alpha_{t+1}, \alpha_{1+2}, \ldots, \alpha_{k}$ sont rationnels.

(b) aucune puissance non nulle de $\exp \left(2 i \pi l ! \alpha_{l}\right)$ n'est une valeur propre de $\mathscr{S}$.

On a alors: pour tout point $\omega$ générique dans $\mathscr{S}$, pour toute fonction $f$ continue sur $\Omega$,

$$
\lim _{N \rightarrow+x} \frac{1}{N} \sum_{n=0}^{N-1}\left\{\exp \left[2 i \pi\left(n \alpha_{1}+n^{2} \alpha_{2}+\cdots+n^{k} \alpha_{k}\right)\right] \cdot f\left(T^{n} \omega\right)\right\}=0 \text {. }
$$

\subsection{Preuve du Théorème 4}

Nous examinons en premier lieu le cas où $l=k$. Rappelons une construction de Furstenberg ([4], chapitre 3). On pose $\alpha=k ! \alpha_{k}$. On considère le système dynamique $\mathscr{S}_{k}=\left((\mathbb{R} / \mathbb{Z})^{k}, m_{k}, S_{k}\right)$, où $m_{k}$ est la mesure de Lebesgue et $S_{k}$ est la transformation définie par

$$
S_{k}\left(x_{1}, x_{2}, \ldots, x_{k}\right)=\left(x_{1}+\alpha, x_{2}+x_{1}, x_{3}+x_{2}, \ldots, x_{k}+x_{k-1}\right) .
$$

Le nombre $\alpha$ étant supposé irrationnel, on sait que $\mathscr{S}_{k}$ est ergodique et même uniquement ergodique, autrement dit que tout point de $(\mathbb{R} / \mathbb{Z})^{k}$ est générique dans $\mathscr{S}_{k}$.

Posons

$$
\begin{aligned}
P_{k}(x) & =\alpha_{1} x+\alpha_{2} x^{2}+\cdots+\alpha_{k} x^{k}, \\
P_{k-1}(x) & =P_{k}(x+1)-P_{k}(x), \\
P_{k-2}(x) & =P_{k-1}(x+1)-P_{k-1}(x), \ldots \\
P_{0}(x) & =\alpha .
\end{aligned}
$$

Chaque $P_{j}$ est un polynôme de degré $j$ et

$$
S_{k}^{\prime \prime}\left(P_{1}(0), P_{2}(0), \ldots, P_{k}(0)\right)=\left(P_{1}(n), P_{2}(n), \ldots, P_{k}(n)\right) .
$$


On considère la fonction $g$ continue sur $(\mathbb{R} / \mathbb{Z})^{k}$, définie par

$$
g\left(x_{1}, x_{2}, \ldots, x_{k}\right)=\exp 2 i \pi x_{k} \text {. }
$$

On a alors

$$
g\left(S_{k}^{n}\left(P_{1}(0), P_{2}(0), \ldots, P_{k}(0)\right)\right)=\exp \left[2 i \pi\left(n \alpha_{1}+n^{2} \alpha_{2}+\cdots+n^{k} \alpha_{k}\right)\right]
$$

Le système $\mathscr{S}_{k}$ est construit à partir du système trivial par une succession finie d'extensions isométriques. Grâce au Théorème 3, on peut affirmer que $\mathscr{S}$ et $\mathscr{P}_{k}$ seront disjoints dès que leur produit sera ergodique. Ces deux systèmes étant ergodiques, il suffit, pour que leur produit soit ergodique, qu'ils n'aient aucune valeur propre en commun (autre que 1). Or les valeurs propres du système $\mathscr{S}_{k}$ sont celles de la rotation $x \mapsto x+\alpha$ sur $\mathbb{R} / \mathbb{Z}$; ce sont les $\exp (2 i \pi p \alpha)$ avec $p \in \mathbb{Z}$. Sous les hypothèses du Théorème 4 , les systèmes $\mathscr{S}$ et $\mathscr{S}_{k}$ sont donc disjoints.

Soit $\omega$ un point générique dans $\mathscr{S}$ et $X_{0}$ un point quelconque dans $(\mathbb{R} / \mathbb{Z})^{k}$. Grâce au Théorème 2 , on peut affirmer que, pour toute fonction $F$ continue $\operatorname{sur}(\mathbb{R} / \mathbb{Z})^{k} \times \Omega$, on a

$$
\lim _{N \rightarrow+\infty} \frac{1}{N} \sum_{n=0}^{N-1} F\left(S_{k}^{n} X_{0}, T^{n} \omega\right)=\int F\left(X, \omega^{\prime}\right) d m_{k}(X) d \mu\left(\omega^{\prime}\right) .
$$

Soit $f$ une fonction continue sur $\Omega$ et $g$ la fonction précédemment définie sur $(\mathbb{R} / \mathbb{Z})^{k}$. En appliquant la formule précédente à la fonction $F=g \otimes f$ et au point $X_{0}=\left(P_{1}(0), P_{2}(0), \ldots, P_{k}(0)\right)$, on obtient exactement le résultat annoncé:

$$
\lim _{N \rightarrow+\infty} \frac{1}{N} \sum_{n=0}^{N-1}\left\{\exp \left[2 i \pi\left(n \alpha_{1}+n^{2} \alpha_{2}+\cdots+n^{k} \alpha_{k}\right)\right] \cdot f\left(T^{n} \omega\right)\right\}=0 .
$$

Le résultat général, dans le cas où $l \in[1, k[$, se déduit du résultat précédent.

Supposons en effet $\alpha_{l+1}, \alpha_{l+2}, \ldots, \alpha_{k}$ rationnels. Si $q$ est un dénominateur commun à ces $k-l$ nombres rationnels, la suite

$$
\exp \left[2 i \pi\left(\alpha_{l+1} n^{l+1}+\alpha_{1+2} n^{l+2}+\cdots+\alpha_{k} n^{k}\right)\right]
$$

est périodique et de période $q$.

On peut alors écrire

$$
\begin{aligned}
& \frac{1}{N} \sum_{n=0}^{N-1} \exp \left[2 i \pi\left(n \alpha_{1}+n^{2} \alpha_{2}+\cdots+n^{k} \alpha_{k}\right)\right] \cdot f\left(T^{n} \omega\right) \\
& =\frac{1}{q} \sum_{p=0}^{q-1}\left(\frac{q}{N} \sum_{\substack{0<n<N \\
n=p[q]}}\left\{\exp \left[2 i \pi\left(n \alpha_{1}+\cdots+n^{\prime} \alpha_{l}\right)\right] \cdot f\left(T^{n} \omega\right)\right\}\right. \\
& \left.\quad \cdot \exp \left[2 i \pi\left(p^{l+1} \alpha_{i+1}+\cdots+p^{k} \alpha_{k}\right)\right]\right)
\end{aligned}
$$

Or, grâce au résultat précédemment démontré on peut affirmer que si $f$ est une fonction continue sur $\Omega$ et si $\omega$ est un point générique dans $\mathscr{S}$, alors

$$
\lim _{N \rightarrow+\infty} \frac{q}{N} \sum_{\substack{0 . n<N \\ n=p[q]}}\left\{\exp \left[2 i \pi\left(n \alpha_{1}+\cdots+n^{\prime} \alpha_{l}\right)\right] \cdot f\left(T^{n} \omega\right)\right\}=0 .
$$

Ceci achève la démonstration du Théorème 4 . 


\subsection{Remarque}

Pour démontrer le Théorème 1 , nous aurons besoin du résultat plus faible suivant.

Proposition 1. Soient $(\Omega, \mu, T)$ un système dynamique et $\alpha_{1}, \alpha_{2}, \ldots, \alpha_{k}$ des nombres réels; alors, pour toute fonction $f$ intégrable sur $(\Omega, \mu)$ et pour $\mu$-presque tout $\omega$, la suite (1) est convergente.

On pourrait justifier cette proposition en invoquant un théorème ergodique récent dû à J. Bourgain [1] et concernant la convergence presque sûre d'expressions de la forme

$$
\frac{1}{N} \sum_{n=0}^{N-1} T_{1}^{P_{1}(n)} T_{2}^{P_{2}(n)} \cdots T_{k}^{P_{k}(n)} F
$$

où les $T_{j}$ sont des transformations qui commutent et préservent la mesure, où les $P_{j}$ sont des polynômes à coefficients entiers et où $F$ est une fonction de carré intégrable.

Mais nous pouvons démontrer cette proposition sans utiliser ce théorème difficile. En fait, on va raisonner par récurrence et la proposition 'à l'ordre $k$ ' sera une conséquence du Théorème 1 'à l'ordre $k-1$ '.

\subsection{Preuve du Théorème 1}

Commençons par remarquer qu'il suffit, pour démontrer le Théorème 1, de prouver le résultat pour tout entier naturel $k$ fixé (il n'y aura ensuite qu'à considérer une réunion dénombrable d'ensembles négligeables). Raisonnons par récurrence sur $k$. Pour $k=0$, c'est le théorème ergodique de Birkhoff. Soit $k \in N^{*}$. Supposons que: pour tout système dynamique $(\Omega, \mu, T)$, pour toute fonction $f$ intégrable $\operatorname{sur}(\Omega, \mu)$, pour $\mu$-presque tout $\omega$, pour tous $\beta_{1}, \beta_{2}, \ldots, \beta_{k-1}$ réels, la suite

$$
\frac{1}{N} \sum_{n=0}^{N-1}\left\{\exp \left[2 i \pi\left(n \beta_{1}+n^{2} \beta_{2}+\cdots+n^{k-1} \beta_{k-1}\right)\right] \cdot f\left(T^{n} \omega\right)\right\}
$$

est convergente. Nous allons déduire de cette hypothèse la proposition suivante.

Proposition 2. Soient $(\Omega, \mu, T)$ un système dynamique et $\alpha$ un nombre réel; alors, pour toute fonction $f$ intégrable sur $(\Omega, \mu)$, pour $\mu$-presque tout $\omega$, pour tous $\alpha_{1}, \alpha_{2}, \ldots, \alpha_{k-1}$ réels, la suite

$$
\frac{1}{N} \sum_{n=0}^{N-1}\left\{\exp \left[2 i \pi\left(n \alpha_{1}+\cdots+n^{k-1} \alpha_{k-1}+n^{k} \alpha_{k}\right)\right] \cdot f\left(T^{n} \omega\right)\right\}
$$

est convergente.

Fixons le système $(\Omega, \mu, T)$ et le réel $\alpha$. Considérons le système $\mathscr{S}_{k}=\left((\mathbb{R} / \mathbb{Z})^{k}, m_{k}, S_{k}\right)$ défini dans la preuve du Théorème 4 . On a

$$
S_{k}\left(x_{1}, x_{2}, \ldots, x_{k}\right)=\left(x_{1}+\alpha, x_{2}+x_{1}, \ldots, x_{k}+x_{k-1}\right),
$$

et, pour tout entier $n>0$, la dernière composante de $S_{k}^{n}\left(x_{1}, x_{2}, \ldots, x_{k}\right)$ est

$$
x_{k}+C_{n}^{1} x_{k-1}+C_{n}^{2} x_{k-2}+\cdots+C_{n}^{k-1} x_{1}+C_{n}^{k} \alpha \text {; }
$$


cette dernière composante s'écrit donc sous la forme $\left(n^{k} / k !\right) \alpha+p(n)$ où $p$ est un polynôme de degré $k-1$.

Appliquons l'hypothèse de récurrence au système produit $\mathscr{S}_{k} \times \mathscr{S}$. On a:

pour toute fonction $F$ intégrable sur $(\mathbb{R} / \mathbb{Z})^{k} \times \Omega$,

pour $m_{k} \otimes \mu$-presque tout $(X, \omega)$ dans $(\mathbb{R} / \mathbb{Z})^{k} \times \Omega$,

pour tous $\beta_{1}, \beta_{2}, \ldots, \beta_{k-1}$ réels,

la suite

$$
\frac{1}{N} \sum_{n=0}^{N-1}\left\{\exp \left[2 i \pi\left(n \beta_{1}+\cdots+n^{k-1} \beta_{k-1}\right)\right] \cdot F\left(S_{k}^{n} X, T^{n} \omega\right)\right\}
$$

est convergente.

Soit $f$ une fonction intégrable sur $(\Omega, \mu)$. Si $X=\left(x_{1}, x_{2}, \ldots, x_{k}\right) \in(\mathbb{R} / \mathbb{Z})^{k}$ et $\omega \in \Omega$, posons

$$
F(X, \omega)=\exp \left(2 i \pi k ! x_{k}\right) \cdot f(\omega)
$$

De (2), on déduit que: il existe $X$ dans $(\mathbb{R} / \mathbb{Z})^{k}$ tel que,

pour $\mu$-presque tout $\omega$,

pour tous $\beta_{1}, \beta_{2}, \ldots, \beta_{k-1}$ réels,

la suite

$$
\frac{1}{N} \sum_{n=0}^{N-1}\left\{\exp \left[2 i \pi\left(n \beta_{1}+\cdots+n^{k-1} \beta_{k-1}\right)\right] \cdot F\left(S_{k}^{n} X, T^{n} \omega\right)\right\}
$$

est convergente.

Fixons un tel point $X=\left(x_{1}, x_{2}, \ldots, x_{k}\right)$. On a

$$
F\left(S_{k}^{n} X, T^{n} \omega\right)=\exp \left[2 i \pi\left(n^{k} \alpha+k ! p(n)\right)\right] \cdot f\left(T^{n} \omega\right) .
$$

De (3), on déduit que: pour $\mu$-presque tout $\omega$, pour tout polynôme $q$ de degré $k-1$, la suite

$$
\frac{1}{N} \sum_{n=0}^{N-1}\left\{\exp \left[2 i \pi\left(n^{k} \alpha+q(n)\right)\right] \cdot f\left(T^{n} \omega\right)\right\}
$$

est convergente. La Proposition 2 est ainsi démontrée.

Etablissons à présent le Théorème 1 (à l'ordre $k$ ). Soient $\mathscr{S}=(\Omega, \mu, T$ ) un système dynamique ergodique et $f$ une fonction continue sur $\Omega$. Notons $\Omega^{\prime}$ l'ensemble des points génériques dans $\mathscr{F}$; l'ergodicité de ce système assure que $\mu\left(\Omega^{\prime}\right)=1$. Pour tout $\alpha$ réel, notons $\Omega_{r}^{\prime}$ l'ensemble des points $\omega$ de $\Omega$ tels que, pour tous $\alpha_{1}, \alpha_{2}, \ldots, \alpha_{k-1}$ réels, la suite

$$
\frac{1}{N} \sum_{n=0}^{N-1}\left\{\exp \left[2 i \pi\left(n \alpha_{1}+\cdots+n^{k-1} \alpha_{k-1}+n^{k} \alpha\right)\right] \cdot f\left(T^{n} \omega\right)\right\}
$$

est convergente. La Proposition 2 affirme que $\mu\left(\Omega_{c}^{\prime}\right)=1$.

Notons $D$ l'ensemble des nombres réels $\alpha$ qui sont rationnels ou tels qu'une puissance non nulle de $\exp [2 \pi i \alpha]$ soit une valeur propre de $\mathscr{S}$. L'ensemble $D$ est 
dénombrable. Le Théorème 4 permet d'affirmer que, pour tout $\alpha$ n'appartenant pas à $D$, on a $\Omega^{\prime} \subset \Omega_{\alpha}^{\prime}$. Posons

$$
\Omega^{\prime \prime}=\left(\bigcap_{\alpha \in D} \Omega_{\alpha}^{\prime}\right) \cap \Omega^{\prime}
$$

On a $\mu\left(\Omega^{\prime \prime}\right)=1$ et, pour tout $\omega$ dans $\Omega^{\prime \prime}$, pour tous $\alpha_{1}, \alpha_{2}, \ldots, \alpha_{k}$ réels, la suite (1) est convergente. Le Théorème 1 (à l'ordre $k$ ) est donc démontré quand $f$ est continue et quand le système dynamique est ergodique.

Le résultat s'étend par densité à toutes les fonctions $f$ intégrables; en effet, si

$$
M(f)=\sup _{\substack{N>0 \\ \alpha_{1}, \ldots, \alpha_{h} \in \mathbb{R}}}\left|\frac{1}{N} \sum_{n=0}^{N-1}\left\{\exp \left[2 i \pi\left(n \alpha_{1}+\cdots+n^{k} \alpha_{k}\right)\right] \cdot f\left(T^{n} \omega\right)\right\}\right|,
$$

l'inégalité maximale de Hopf assure que, pour tout $\lambda>0$,

$$
\mu(\{M(f)>\lambda\}) \leq \frac{\|f\|_{1}}{\lambda} ;
$$

cette inégalité maximale uniforme en les $\alpha_{j}$, entraîne que l'ensemble des fonctions $f$ vérifiant la conclusion du Théorème 1 est feimé dans $L^{1}(\mu)$.

Pour achever la démonstration il ne reste plus qu'à ôter l'hypothèse d'ergodicité du système dynamique. Ceci se fait par le procédé usuel de désintégration d'une probabilité invariante en barycentre de probabilités ergodiques. En effet cette formule de désintégration permet d'affirmer que: si une partie mesurable $\Omega$ " de $\Omega$ vérifie $\mu\left(\Omega^{\prime \prime}\right)=1$ pour toute probabilité $\mu$ qui est $T$-invariante et ergodique, alors on a $\mu\left(\Omega^{\prime \prime}\right)=1$ pour toute probabilité $\mu$ qui est $T$-invariante.

La preuve du Théorème 1 (à l'ordre $k$ ) est terminée. Ceci achève le raisonnement par récurrence et la démonstration du Théorème 1.

\section{REFERENCES}

[1] J. Bourgain. Théorèmes ergodiques ponctuels pour certains ensembles arithmétiques. Note au $C$. R. Acad. Sci. Paris 305 (1987), 397-402.

[2] H. Furstenberg. Strict ergodicity and transformations of the torus. Amer. J. Math. 83 (1961), 573-601.

[3] H. Furstenberg. Disjointness in ergodic theory, minimal sets, and a problem in diophantine approximation. Math. Systems Theory 1 (1967), 1-49.

[4] H. Furstenberg. Recurrence in Ergodic Theory and Combinatorial Number Theory. Princeton University Press: 1981.

[5] E. Lesigne. Théorèmes ergodiques pour une translation sur une nil-variété. Ergod. Th. \& Dynam. Sys. 9 (1989), 115-126.

[6] J. P. Thouvenot. La démonstration de Furstenberg du théorème de Szemerédi sur les progressions arithmétiques. Séminaire Bourbaki (1977/78) no. 518.

[7] N. Wiener \& A. Wintner. Harmonic analysis and ergodic theory. Amer. J. Math. 63 (1941), 415-426. 